\title{
Application of multi-dimensional grouping to building steel stiffened shell structures
}

\author{
Remigiusz Iwańkowicz, Ph.D. \\ The West Pomeranian University of Technology, Szczecin, Poland
}

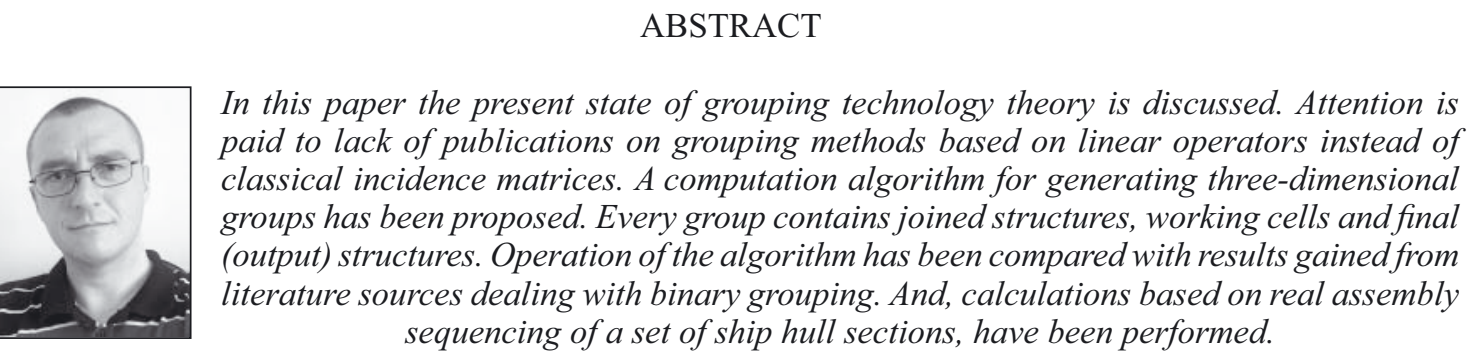

Keywords: group technology; assembly sequencing; steel structures manufacturing

\section{INTRODUCTION}

Hulls of typical merchant ships belong to a group of steel stiffened shell structures (3S). Such structures can be found also in very large tanks, bridge elements, offshore units etc. Because of their dimensions and necessity to expand working frontage, production of $3 \mathrm{~S}$ structures is usually carried out in accordance with the so called prefabrication subdivision. It means that final product is composed of many simultaneously produced prefabricated units (subassemblies). They usually are further subdivided into lower stage units. Consequently, a multistage assembly sequencing is formed. Number of stages depends on structure geometry and manufacturing conditions of a producer (a. o. - cranes at his disposal). $3 \mathrm{~S}$ structures are as a rule produced in a few pieces or short series, that forces enterprises such as shipyards to keep agile production organisation and makes designing and production preparation work more labour consuming. The problem of a degree of producing in series is tightly connected with the notion of technological similarity of products. Products even if different visually and functionally may require similar production processes and - from the enterprise point of view - belong to one common series.

One of the cardinal tasks of design office is to act in accordance with the principle of ,design for production" by applying as much standardization of design solutions as possible, including assortment of rolled profiles and shapes of cut details. Such actions are of a low degree of structural complexity.

A successive action aimed at minimization of unitary production character of $3 \mathrm{~S}$ structures is searching for such division planes for their assembly as to obtain the effect of a greater degree of serial production at least in early stages of assembly process. Two $3 \mathrm{~S}$ structures may differ from each other in technology, for instance: by accessibility for automative welders, shapes of curvatures, assembling sequence and possibility of being manoeuvered. Simultaneously, their structurally simpler subassemblies may be very similar stiffened panels as regards their technology.

The theory which makes it possible, on the level of models, to:

- measure technological similarity,

- state when a given product can be qualified to be an element of greater series, as well as

- so design a given production system as it could realize production in a way as repeatable as possible

is the classical theory of group technologies (GT). Its main aim is to distiguish, in the set of resources used in the process or set of products, some subsets called groups. In the literature $[2,3,4,5,13]$ only working cells of different kinds or, simply, machines are usually distinguished as grouped resources. In the case of grouping the products we aim at maximization of their repeatability and reduction of design and production preparation processes [11]. Due to having at hand certain groups of products, designers of prototypes have at their disposal hints helping to maintain high producibility.

Among profits resulting from application of group technologies to organizing production cells the following are numbered [9]:

- widened standardization of working cells, applied instrumentation, set-ups of working parameters, 
- reduction of interoperative transport as a result of relocation of work objects within production cells,

- facilitated scheduling of production,

- shortened cycles of production,

- lessened buffer stores and lowered freeze level of current assets,

- elevated motivation of employees and simplified procedures of worktime estimating.

The grouping is performed with use of:

- visual assessment of elements of a set - on the basis of a subjective validation by experts,

- classification and coding - on the basis of an assumed coding system of features (in the case of products: their geometry and production technology),

analysis of technological routes realized by products.

The method of analyzing technological routes is advantagous as it makes it possible to group simultaneously a set of resources and products. Here the groups of the set of resources are called input ones.

Identification of input and output groups permits also to design the so called production cells where each of them constitutes the pair (group of resources; group of products). Such pairs are called two-dimensional. Algorithms for twodimensional grouping have been developed for a few dozen years $[6,1,16]$.

The grouping of factors by using the method of analyzing technological routes is realized according to the following scheme [16]:

1. The formulating of relations between resources and products

by using the so called incidence matrix - to each of the resources an appropriate row of the matrix is assigned, and to each of the products - a column of it (or vice versa - it is a question of convention).

2. The determining of mutual technological similarities between resources as well as between products. Consequently, two matrices of technological similarities are built - separately for each of the analyzed sets.

3. The setting of a threshold for technological similarity, above which it has to be considered that two elements of the set in question should be assigned to the same group.

The two-dimensional grouping makes it possible to respond quickly to the question: how to organize production cells in the system if planned assortment of products and engineering techniques available to producer, are known? It is easy to reach the effect if the incidence matrix unambigously describes relations between given products and working cells. However in practice the forming of such incidence matrix is very labourconsuming and demanding comprehensive knowledge on technologies used in a given enterprise.

An extension of the classical two-dimensional grouping method is the proposal of distinguishing, in the set of used resources, two separable subsets. As a result, we have to do with three sets and perform analysis of similarity between products on the basis of their incidence with the first and second set of resources. In both the sets of resources we analyze similarity independently, on the basis of incidence with the second subset of resources as well as the set of products. From the analysis result three-dimensional groups, i.e. the ordered threes: (1 ${ }^{\text {st }}$ group of resources, $2^{\text {nd }}$ group of resources, group of products). The first concepts of the kind belong to Min and Shin [17]. Their proposals deal with analysis of technological similarities in the two subsets of resources: machines and operators as well as in the set of products. Successive elaborations have been a natural generalization towards $n$-dimensional models $[14,15,18]$.

\section{CONCEPT OF THREE-DIMENSIONAL GROUPING IN MANUFACTURING 3S STRUCTURES}

\subsection{Input data, standardizing and scaling}

The three-dimensional grouping is proposed for application in manufacturing $3 \mathrm{~S}$ structures with taking into account an ordered set of various working cells in which joining process of structures into higher stage structural units, is performed.

The following notions are introduced:

- $\mathrm{S}=\left\{\mathrm{s}_{1}, \ldots, \mathrm{s}_{\mathrm{m}}\right\} \quad$ - working cells,

- $\quad C^{\text {in }}=\left\{c_{1}^{\text {in }}, \ldots, c_{n}^{\text {in }}\right\} \quad$ - joined structures (put in the cells),

- $\quad C^{\text {out }}=\left\{c_{1}^{\text {out }}, \ldots, c_{p}^{\text {out }}\right\}-$ produced structures (put out the cells).

The structures differ to each other as regards their geometry. And, it is not essential where butting contacts of plate sheets are placed, nor other features of technological importance (e.g. form of cut-outs and gasket plates). Only the functional features are taken into account, namely: arrangement of shells, stiffeners and brackets, ways of ensuring continuity of girders etc. Structures of various complexity degrees are considered. It may be single details or large-size spatial structures, e.g. hull block sections or even entire ship hull.

To every output structure can be assigned elements of the set $\mathrm{S}$ and $\mathrm{C}$ in which take part in its production. To every working cell can be assigned structures which are delivered to it. It leads to formulation of three matrices of incidence between relevant sets. The concepts have been published so far are based on binary matrices. However production of $3 \mathrm{~S}$ structures is associated with a rather low diversity of cells. And, differences between structures amount to labour consumption of processes as well as quantity of lower-stage subassemblies of which the structures are formed. Practical implementation of grouping technologies into production of $3 \mathrm{~S}$ structures faces the problem of an insufficient informing force of binary incidence matrices. For instance, structures which greatly differ to each other as regards requirements for equipment of working cells, can be built of identical subassemblies and with the use of identical welding methods. In this case, application of the binary grouping methods may lead to erroneous placing technologically different products into the very same cells. As a result low specialized production cells are obtained, that contradicts their essence.

As a solution for the above mentioned problems it is proposed to apply a grouping model based on continuous incidence matrices. For every pair of sets of the input factors $\mathrm{X}$ and output factors $\mathrm{Y}$ the following matrix is determined: $\mathrm{A}^{\mathrm{X}-\mathrm{Y}} \in \mathrm{M}\left(|\mathrm{X}| \times|\mathrm{Y}|, \mathbb{R}^{+} \cup\{0\}\right)$, where $\mathrm{a}_{\mathrm{ij}}^{\mathrm{X}-\mathrm{Y}}>0$, if production of $j$-th element of the set $Y$ requires to angage $i$-th element of the set $X$, if not: $a_{i j}^{X-Y}=0$. Values of the non-zero coefficients $a_{i j}^{X-Y}$ are standards of consumption of input factors per production unit of appropriate output factors. In the case of working cells the consumption standards usually represent the following:

- amount of working time of a cell during production of unit of a structure,

- amount of a concrete effect of work of a cell, generated during production of unit of a structure (e.g. one meter of welded joint, one square meter of painted surface etc).

The continuous incidence matrix is a linear operator acting, in this case, between vector spaces of production factors [12]. Such operators find wide application to a.o. operational research, and they may find much wider use in solving the grouping problems in enterprise. 


\subsection{Measure of grouping quality}

Three-dimensional grouping the sets $\mathrm{S}, \mathrm{C}^{\text {in }}$ and $\mathrm{C}^{\text {out }}$ is an optimization problem and amounts to searching for the division $\Gamma$ of the Cartesian product $\mathrm{S} \times \mathrm{C}$ in $\times$ Cout into $\gamma$ subsets:

$$
\begin{aligned}
& \Gamma=\Gamma\left(S \times C^{\text {in }} \times C^{\text {out }}, \gamma\right)= \\
& =\left\{\Gamma_{i}=\left(S_{i}, C_{i}^{\text {in }}, C_{i}^{\text {out }}\right) \in S \times C^{\text {in }} \times C^{\text {out }}:(i=1, \ldots, \gamma), \bigcap_{i=1}^{\gamma} S_{i}=\bigcap_{i=1}^{\gamma} C_{i}^{\text {in }}=\bigcap_{i=1}^{\gamma} C_{i}^{\text {out }}=\varnothing\right\}
\end{aligned}
$$

The groups $\Gamma_{i}$ are really sums of the subsets $S_{i}, C_{i}$ in and $C_{i}$ out, however they are written in the form of ordered threes, that facilitates to identify elements of each of the group.

The division $\Gamma$ can be assessed as regards its quality. In 1986 Chandrasekharan and Rajagopalan [7] proposed to apply a criterion for the two-dimensional grouping of binary incidence matrix. This is maximization of a weighted sum of densities of submatrices and their neighborhood. And, the density is meant to be number of unities related to whole number of elements of the matrix in question. In $2007 \mathrm{Li}$ [15] extended the criterion, for the multi-dimensional case, by assigning weights to particular incidence matrices.

In the case of continuous matrices, differences in values of the elements should be accounted for additionally apart from the fact of occurrence of non-zero elements of incidence matrices in the space of groups and outside it.

Let the weights of particular incidence matrices be equal to: $\omega_{\mathrm{S}-\mathrm{C}_{\mathrm{in}}}, \omega_{\mathrm{S}-\mathrm{C}_{\mathrm{out}}}, \omega_{\mathrm{C}_{\mathrm{in}}-\mathrm{C}_{\text {out }}}$ respectively. Then it is proposed to take, as a quality measure for $\Gamma$ division, the following criterion:

$$
E(\Gamma)=\frac{\omega_{S-C_{\text {in }}} \cdot W\left(A^{S-C^{\text {in }}}, \Gamma\right)+\omega_{S-C_{\text {out }}} \cdot W\left(A^{S-C^{\text {out }}}, \Gamma\right)+\omega_{C_{\text {in }}-C_{\text {out }}} \cdot W\left(A^{C^{\text {in }}-C^{\text {out }}}, \Gamma\right)}{\omega_{S-C_{\text {in }}}+\omega_{S-C_{\text {out }}}+\omega_{C_{\text {in }}-C_{\text {out }}}} \rightarrow \max
$$

where:

$\mathrm{W}\left(\mathrm{A}^{\mathrm{X}-\mathrm{Y}}\right)$ - grouping quality index for continuous matrix of incidence between the set $\mathrm{X}$ and $\mathrm{Y}$.

Let the set of indices of elements of the set $\mathrm{X}$ belonging to k-th group is marked $\mathrm{I}_{\mathrm{k}}^{\mathrm{X}}$. Then the following is defined:

$$
W\left(A^{X-Y}, \Gamma\right)=q \cdot \eta_{1}\left(A^{X-Y}, \Gamma\right)+(1-q) \cdot \eta_{0}\left(A^{X-Y}, \Gamma\right)
$$

where:

$\eta_{1}\left(A^{X-Y}, \Gamma\right)-$ sum of values of elements of the matrix $A^{X-Y}$ belonging to all groups, related to their total number,

$\eta_{0}\left(A^{X-Y}, \Gamma\right)-$ sum of values of elements of the matrix $A^{X-Y}$ not belonging to any of the groups, related to their total number,

$\mathrm{q}-\quad-$ weight of membership of elements in groups in relation to their possible being left outside the groups. In the literature $\mathrm{q}=0.5$ is often assumed [15].

$$
\begin{gathered}
\eta_{1}\left(A^{X-Y}, \Gamma\right)=\frac{\sum_{k=1}^{\gamma} \sum_{i \in I_{k}^{X}} \sum_{j \in I_{k}^{Y}} a_{i j}^{X-Y}}{\sum_{k=1}^{\gamma}\left|I_{k}^{X}\right| \cdot\left|I_{k}^{Y}\right|} \\
\eta_{0}\left(A^{X-Y}, \Gamma\right)=\frac{\sum_{k} \sum_{l \neq k} \sum_{i \in I_{k}^{X}} \sum_{j \in I_{l}^{Y}} a_{i j}^{X-Y}}{\sum_{k} \sum_{l \neq k}\left|I_{k}^{X}\right| \cdot\left|I_{l}^{Y}\right|}
\end{gathered}
$$

The quantity $\eta_{1}\left(\mathrm{~A}^{\mathrm{X}-\mathrm{Y}}, \Gamma\right)$ is the mean value of all elements within groups, and $\eta_{0}\left(\mathrm{~A}^{\mathrm{X}-\mathrm{Y}}, \Gamma\right)$ is the mean value of elements outside the groups. As the elements $\mathrm{a}_{\mathrm{ij}}^{\mathrm{X}-\mathrm{Y}}$ are normalized within the range [0,1], both the quantities are also contained in this interval, consequently, the inequalities: $0 \leq \mathrm{W}\left(\mathrm{A}^{\mathrm{X}}-\mathrm{Y}, \Gamma\right) \leq 1,0 \leq \mathrm{E}(\Gamma) \leq 1$ are also satisfied.

Additionally, every group is assumed to be consisted of sequence of non-empty sets. For instance, to the groups cannot be assigned only a set of output structures and cells without a set of input structures. The assumption is aimed at preventing against natural grouping input structures into one group, cells into second group and output structures into third group.

\subsection{Algorithm of the three-dimensional grouping for continuous incidence matrices}

The algorithm of multi-dimensional grouping was presented by Li in 2003 [14]. However this proposal is limited to binary incidence matrices. As a result it does not make use of cluster analysis methods but is the author's solution strongly based on the binary description of elements of sets.

Below are presented principal steps of the proposed algorithm. It is of a recurrential character. The grouping is realized by determining centres of groups having assumed number of elements and assigning to them the closest elements according to a selected measure. 
The requirement of that every group should contain elements from all the grouped sets is satisfied in Step 2.5, in which one element of each of the sets is assigned to each of the groups and only in the next step unlimited assignment of remaining elements takes place.

As already mentioned, it is worth normalizing the continuous incidence matrices within a selected interval.

\section{Start-up of the algorithm}

\section{Step 1:}

Having at our disposal normalized continuous matrices of incidence between the sets: $\mathrm{S}, \mathrm{C}^{\text {in }}$ and $\mathrm{C}^{\text {out }}$ we form the following integrated matrix:

$$
A=\left(\begin{array}{c|c|c}
0 & A^{S-C^{\text {in }}} & A^{S-C^{\text {out }}} \\
\hline\left(A^{S-C^{\text {in }}}\right)^{T} & 0 & A^{C^{\text {in }}-C^{\text {out }}} \\
\hline\left(A^{S-C^{\text {out }}}\right)^{T} & \left(A^{C^{\text {in }}-C^{\text {out }}}\right)^{T} & 0
\end{array}\right)_{(m+n+p) \times(m+n+p)}
$$

Further proceeding consists in solving the problem of two-dimensional grouping, where the cells and input and output structures are considered jointly as the so called set of elements:

$$
F=S \cup C^{\text {in }} \cup C^{\text {out }}=\left\{f_{1}, \ldots, f_{m+n+p}\right\}
$$

The elements numbered from 1 to $\mathrm{m}$ represent working cells, the elements of numbers from $m+1$ to $m+n-$ input structures and the elements of numbers from $m+n+1$ to $m+$ $n+p-$ output structures. Therefore we have:

$$
\begin{aligned}
& f_{1}=s_{1}, \ldots, f_{m}=s_{m}, \\
& f_{m+1}=c_{1}^{\text {in }}, \ldots, f_{m+n}=c_{n}^{\text {in }}, \\
& f_{m+n+1}=c_{1}^{\text {out }}, \ldots, f_{m+n+p}=c_{p}^{\text {out }}
\end{aligned}
$$

\section{Step 2:}

The division $\mathrm{K}$ of a set of elements $\mathrm{F}$ into clusters (onedimensional groups) such that one working cell, one input structure and one output structure is present in each of them:

$$
\begin{aligned}
K(F)= & \left\{K_{i} \subset F:\right. \\
& i=1, \ldots, \min \left\{|S|,\left|C^{\text {in }}\right|,\left|C^{\text {out }}\right|\right\}, \\
& \left.\underset{i}{\forall}\left(K_{i} \cap S \neq \varnothing, K_{i} \cap C^{\text {in }} \neq \varnothing, K_{i} \cap C^{\text {out }} \neq \varnothing\right)\right\}
\end{aligned}
$$

The division $\mathrm{K}$ should be performed for the following number of clusters:

$$
\gamma=2, \ldots, \min \left\{|S|,\left|C^{\text {in }}\right|,\left|C^{\text {out }}\right|\right\}
$$

Such division for a given $\gamma$ is marked $\mathrm{K}^{\gamma}$. Successive steps of the algorithm should be made for each of the variant of $\gamma$ value.

The division into clusters can be realized by using one of the divison-based algorithms. Here a modified c-means method is implemented. The method in its classical form has been described a.o. by Hartigan in 1975 [10] as well as Tan, Steinbach and Kumar in 2005 [19]. The proposed modification is aimed at ensuring occurrence, in each of the clusters, at least one element out of each of the sets: $\mathrm{S}, \mathrm{C}$ in and $\mathrm{Cout}$.
Step 2.1:

The forming of the cluster division matrix $\Lambda^{\gamma}=(0)_{\mathrm{q} \times \gamma}$, where $\mathrm{q}=\mathrm{m}+\mathrm{n}+\mathrm{p}$ is total number of factors.

In its successive steps the algorithm assigns value 1 (unity) to components of the division matrix at intersection of cluster columns and rows of relevant factors. The initial division is made in accordance with the following algorithm:

$$
\begin{aligned}
& r \leftarrow\left\lfloor\frac{\min \{m, n, p\}}{\gamma}\right] ; \quad \text { //rounding down } \\
& \text { for }[j=1,2, \ldots, \gamma-1]: \\
& \qquad \begin{array}{l}
\text { for }[i=1+(j-1) \cdot r, \ldots, j \cdot r]: \quad \Lambda_{i j}^{\gamma} \leftarrow 1 ; \\
\text { for }[i=m+1+(j-1) \cdot r, \ldots, m+j \cdot r]: \quad \Lambda_{i j}^{\gamma} \leftarrow 1 ; \quad(2.11) \\
\text { for }[i=m+n+1+(j-1) \cdot r, \ldots, m+n+j \cdot r]: \quad \Lambda_{i j}^{\gamma} \leftarrow 1 ;
\end{array}
\end{aligned}
$$$$
\text { for }[i=1+(\gamma-1) \cdot r, \ldots, m]: \quad \Lambda_{i \gamma}^{\gamma} \leftarrow 1 \text {; }
$$$$
\text { for }[i=m+1+(\gamma-1) \cdot r, \ldots, m+n]: \quad \Lambda_{i j}^{\gamma} \leftarrow 1 \text {; }
$$$$
\text { for }[i=m+n+1+(\gamma-1) \cdot r, \ldots, q]: \quad \Lambda_{i j}^{\gamma} \leftarrow 1 \text {; }
$$

\section{return $\Lambda^{\gamma}$;}

Step 2.2:

The determining of the matrix of cluster centres, $\left(\alpha_{\mathrm{ij}}\right)_{\mathrm{q} \times \gamma}$ :

$$
\underset{j=1, \ldots, \gamma}{\forall} \alpha^{\langle j\rangle}=\frac{\sum_{i=1}^{q} A^{\langle i\rangle} \cdot \Lambda_{i j}^{\gamma}}{\sum_{i=1}^{q} \Lambda_{i j}^{\gamma}}
$$

Step 2.3:

The determining of the matrix of distances between elements and cluster centres.

For every $\mathrm{i}$-th element and $\mathrm{j}$-th group, $\mathrm{D}_{\mathrm{ij}}$ value is determined. Here, it is possible to assume an arbitrary measure, not necessarily metric one. The following variants are proposed to be considered:

- angular measure (scalar product of vectors):

$$
\underset{i=1, \ldots, q, q j=1, \ldots, \gamma}{\forall} \quad D_{i j}=\frac{A^{\langle i\rangle T} \cdot \alpha^{\langle j\rangle}}{\sqrt{\sum_{k=1}^{q} A_{k i}{ }^{2}} \cdot \sqrt{\sum_{k=1}^{q} \alpha_{k j}{ }^{2}}}
$$

- Manhattan measure:

$$
\underset{i=1, \ldots, q}{\forall} \underset{j=1, \ldots, \gamma}{\forall} \quad D_{i j}=\left|A_{1}^{\langle i\rangle}-\alpha_{1}^{\langle j\rangle}\right|+\ldots+\left|A_{q}^{\langle i\rangle}{ }_{q}-\alpha_{q}^{\langle j\rangle}\right|
$$

- Euclidean measure:

$$
\underset{i=1, \ldots, q}{\forall} \underset{j=1, \ldots, \gamma}{\forall} D_{i j}=\sqrt{\left(A^{\langle i\rangle}-\alpha^{\langle j\rangle}\right)_{1}^{2}+\ldots+\left(A^{\langle i\rangle}-\alpha^{\langle j\rangle}\right)_{q}^{2}}
$$

- Chebyshev measure:

$$
\underset{i=1, \ldots, q}{\forall} \underset{j=1, \ldots, \gamma}{\forall} D_{i j}=\max _{k=1, \ldots, q}\left\{\left|A^{\langle i\rangle}{ }_{k}-\alpha_{k}^{\langle j\rangle}\right|\right\}
$$

- Mahalanobis measure:

$$
\begin{gathered}
\underset{i=1, \ldots, q, q j=1, \ldots, \gamma}{\forall} D_{i j}=\sqrt{\left(A^{\langle i\rangle}-\alpha^{\langle j\rangle}\right)^{T} \cdot V^{-1} \cdot\left(A^{\langle i\rangle}-\alpha^{\langle j\rangle}\right)} \\
V=\operatorname{diag}\left(\sigma_{1}^{2}, \ldots, \sigma_{q}^{2}\right)
\end{gathered}
$$


In the below presented exemplary calculations the above specified measures have been mutually compared as regards their effectiveness.

Step 2.4:

The saving of the cluster division matrix after introducing a new variable: $\hat{\Lambda}=\Lambda$.

The zeroing of the cluster division matrix: $\Lambda \gamma=(0)_{\mathrm{q} \times \gamma}$.

\section{Step 2.5:}

The assigning of one factor from each of the three subsets to every cluster. This way three-element clusters $\gamma$ in number were built. Below is presented the algorithm of assigning the factors numbered from 1 to $\mathrm{m}$, i.e. these belonging to the subset of cells:

$$
\begin{aligned}
& \text { for }[i=1,2, \ldots, m] \text { : } \\
& \text { for }[j=1,2, \ldots, \gamma]: \quad \Delta_{i j} \leftarrow D_{i j} ; \quad \text { //forming local matrix of distances } \\
& \text { for }[l=1,2, \ldots, \gamma] \text { : } \\
& d=\min _{\substack{i=1, \ldots, m \\
j=1, \ldots, \gamma}}\left\{\Delta_{i j}\right\} ; \\
& \text { for }[i=1,2, \ldots, m] \text { : } \\
& \text { for }[j=1,2, \ldots, \gamma] \text { : } \\
& \text { if }\left[\Delta_{i j}=d\right] \text { : } \\
& \Lambda_{i j}^{\gamma} \leftarrow 1 ; \quad \text { //assigning object to cluster } \\
& \Delta^{\langle j\rangle} \leftarrow(\max \{\Delta\}+1)_{m \times 1} ; \quad / / \text { local blocking } j \text {-th cluster (column) } \\
& \Delta^{T\langle i\rangle} \leftarrow(\max \{\Delta\}+1)_{\gamma \times 1} ; / / \text { local blocking } i \text {-th object (row) } \\
& D^{T\langle i\rangle} \leftarrow(\max \{D\}+1)_{\gamma \times 1} ; / / \text { global blocking } i \text {-th object (row) }
\end{aligned}
$$

\section{return $\Lambda^{\gamma}$;}

For the two successive subsets the procedure runs analogously. Changes deal only with the range of variability of factor's number in the loops for $[i=\ldots]$.

\section{Step 2.6:}

In the preceding step it was ensured that in each of the $\gamma$ clusters one working cell, one input structure and one output structure was placed. Now it is possible to assign factors, without any limitations, by taking into account only the criterion of minimization of distances from the cluster centres $\alpha^{\langle\mathrm{j}\rangle}$.

$$
\begin{aligned}
& \operatorname{for}[i=1,2, \ldots, q]: \\
& \quad \operatorname{for}[j=1,2, \ldots, \gamma]: \\
& \quad \operatorname{if}\left[D_{i j}=\min _{j=1, \ldots, \gamma}\left\{D^{T\langle i\rangle}\right\}\right]: \Lambda_{i j}^{\gamma}=1 ; \\
& \text { return } \Lambda^{\gamma} ;
\end{aligned}
$$

Step 2.7:

Comparison between the obtained cluster division matrix and the solution reached from the preceding loop. Here Frobenius norm is applied to difference between the compared matrices:

$$
\left\|\Lambda^{\gamma}-\hat{\Lambda}\right\|_{F}=\sqrt{\sum_{i=1}^{q} \sum_{j=1}^{\gamma}\left(\lambda_{i j}^{\gamma}-\hat{\lambda}_{i j}\right)^{2}}
$$

Further action is dependent on assessment of value of the norm . The assessment is made by comparing its result with the assumed limit value $\delta$ experimentally determined for a given case.

$$
\text { if }\left[\left\|\Lambda^{\gamma}-\hat{\Lambda}\right\|_{F} \leq \delta\right]: \text { go to step } 3 ;
$$

\section{otherwise : go to step 2.2;}

Step 3:

For the determined $\gamma$ the cluster division matrix $\Lambda^{\gamma}$ is known. The clusters $\mathrm{K}_{1}^{\gamma}, \ldots, \mathrm{K}_{\gamma}^{\gamma}$ are also given in the form of sets of the factors to which correspond unity values in respective columns of the matrix $\Lambda^{\gamma}$. As a result the division $\Gamma$ into threedimensional groups is obtained:

$$
\underset{i=1, \ldots, \gamma}{\forall} \Gamma_{i}=\left(K_{i}^{\gamma} \cap S, K_{i}^{\gamma} \cap C^{i n}, K_{i}^{\gamma} \cap C^{\text {out }}\right)
$$

Step 4:

Comparison of variants of number of clusters and applied distance measures is performed by determining the division which maximizes value of the objective function :

$$
\Gamma^{*}=\Gamma: E(\Gamma) \rightarrow \max
$$

\section{The end of the algorithm}

The investigating of many variants of number of clusters may seem time-consuming. However it should be taken into 
account that in practical building $3 \mathrm{~S}$ structures we deal with a total number of different working cells, which amounts to a few dozen only. This is much less than that of possible variants of input and output structures.

The algorithm makes it possible to search for similarities between produced $3 \mathrm{~S}$ structures, working cells and structures which are joined together in the cells. As a result, is obtained information which deals with the following:

- $\quad$ structure of working cells, i.e. which operations have to be realized by the cells and how many working cells should realize given operations,

- kinds of the structures which will be most often delivered to the cells to be joined together there, which is connected with ensuring appropriate transport means, as well as with possible location of the cells and manufacturers of subassemblies in close neighbourhood,

- indication of a cell which has to be responsible for production of a given structure in the case when the structure is qualified to belong to one of the groups.

The described problem may seem to be solvable by independent realization of the two two-dimensional grouping: output structures - cells, input structures - cells. However it should be observed that possibility of building a given output structure by a production cell is strictly dependent on a degree of complexity of input structures which have to be joined together. Two cells may produce the same structure, however one of them may be specialized in assembling complex subassemblies whereas the other may realize the process of assembling single details to form simple structural units. Therefore the output structure will be assigned to three-dimensional group only if it itself shows technological similarity and also the input structures and working cells coincidental with it do the same.

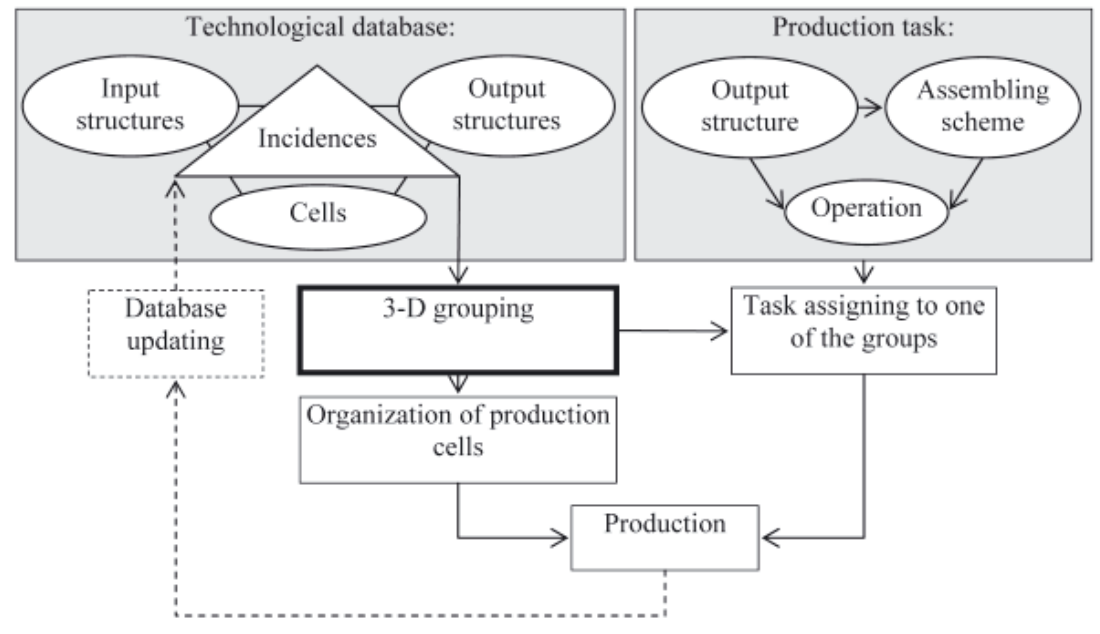

Fig. 2.1. Location of the proposed algorithm into production planning process of $3 S$ structures

\section{EXEMPLARY CALCULATIONS}

\subsection{Example 1 - the binary problem proposed by $\mathrm{Li}$}

In $2007 \mathrm{Li}$ [15] considered the following 3-D binary problem (let's call it: „Li” problem):

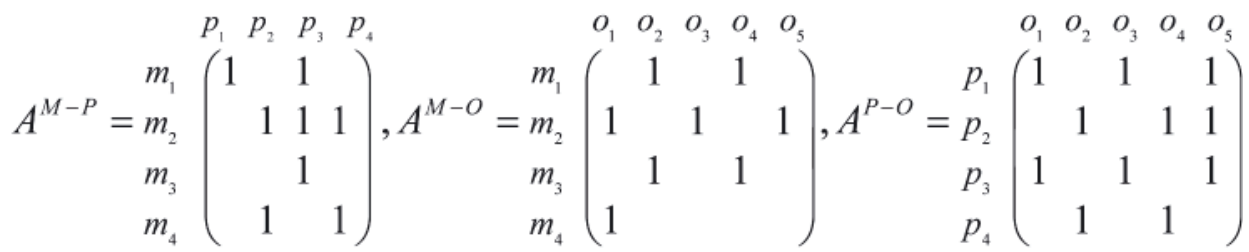

and formulated its pseudo-optimum solution in the form of division into the two groups:

$$
\begin{aligned}
& \Gamma_{1}=\left(\left\{m_{2}, m_{4}\right\},\left\{p_{2}, p_{4}\right\},\left\{o_{1}, o_{3}, o_{5}\right\}\right) \\
& \Gamma_{2}=\left(\left\{m_{1}, m_{3}\right\},\left\{p_{1}, p_{3}\right\},\left\{o_{2}, o_{4}\right\}\right)
\end{aligned}
$$

The grouping can be represented by the sorted incidence matrices with the distinguished submatrices of groups as follows:

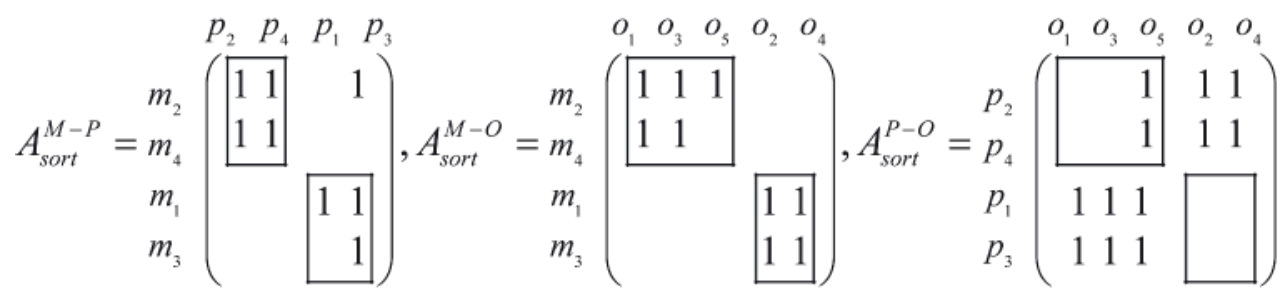


The quality index of the above mentioned grouping, under assumption of the weights $q=0.5$ and $\omega_{1}=\omega_{2}=\omega_{3}$, amounts to: $\mathrm{E}\left(\Gamma^{\mathrm{Li}}\right)=1 / 3(0.875+0.95+0.05)=0.625$.

The Li's solution is compared now with the results obtained by means of the proposed algorithm and distance measures described by Eqs. through. As can be observed in this case the division can be done into 2, 3 or 4 groups. Tab. 3.1 presents the divisions which provide the best values of the quality indices for particular measures.

Tab. 3.1. Results of solving the problem , $L i "$

\begin{tabular}{|c|l|c|}
\hline Measure & \multicolumn{1}{|c|}{ Division } & \multicolumn{1}{|c|}{$\begin{array}{c}\text { Quality } \\
\text { index }\end{array}$} \\
\hline \multirow{2}{*}{ Manhattan } & $\begin{array}{l}\Gamma_{1}=\left(\left\{m_{1}\right\},\left\{p_{4}\right\},\left\{o_{1}\right\}\right), \\
\Gamma_{3}=\left(\left\{m_{2}\right\},\left\{p_{3}\right\},\left\{o_{4}\right\}\right),\end{array}$ & 0.267 \\
& $\Gamma_{3}=\left(\left\{m_{3}\right\},\left\{p_{2}\right\},\left\{o_{3}, o_{5}\right\}\right)$, & \\
\hline Euclidean, & $\Gamma_{4}=\left(\left\{m_{4}\right\},\left\{p_{1}\right\},\left\{o_{2}\right\}\right)$ & $\Gamma_{1}=\left(\left\{m_{2}\right\},\left\{p_{1}, p_{2}, p_{3}\right\},\left\{o_{1}\right\}\right)$, \\
Mahalanobis & $\Gamma_{2}=\left(\left\{m_{1}, m_{3}, m_{4}\right\},\left\{p_{4}\right\},\left\{o_{2}, o_{3}, o_{4}, o_{5}\right\}\right)$ & 0.511 \\
\hline Angular & $\Gamma_{1}=\left(\left\{m_{1}\right\},\left\{p_{1}\right\},\left\{o_{1}\right\}\right)$, & \\
& $\Gamma_{2}=\left(\left\{m_{2}\right\},\left\{p_{2}\right\},\left\{o_{4}\right\}\right)$, & 0.567 \\
& $\Gamma_{3}=\left(\left\{m_{3}\right\},\left\{p_{3}\right\},\left\{o_{3}\right\}\right)$, & \\
& $\Gamma_{4}=\left(\left\{m_{4}\right\},\left\{p_{4}\right\},\left\{o_{2}, o_{5}\right\}\right)$ & \\
\hline \multirow{2}{*}{ Chebyshev } & $\Gamma_{1}=\left(\left\{m_{1}, m_{2}, m_{4}\right\},\left\{p_{1}, p_{2}, p_{3}\right\},\left\{o_{1}, o_{3}, o_{5}\right\}\right)$, & $\mathbf{0 . 6 6 8}$ \\
& $\Gamma_{2}=\left(\left\{m_{3}\right\},\left\{p_{4}\right\},\left\{o_{2}, o_{4}\right\}\right)$ & \\
\hline
\end{tabular}

As can be observed, the Chebyshev measure provides the division of the quality index higher than the solution obtained by Li. The division can be represented by the following sorting of incidence matrices:

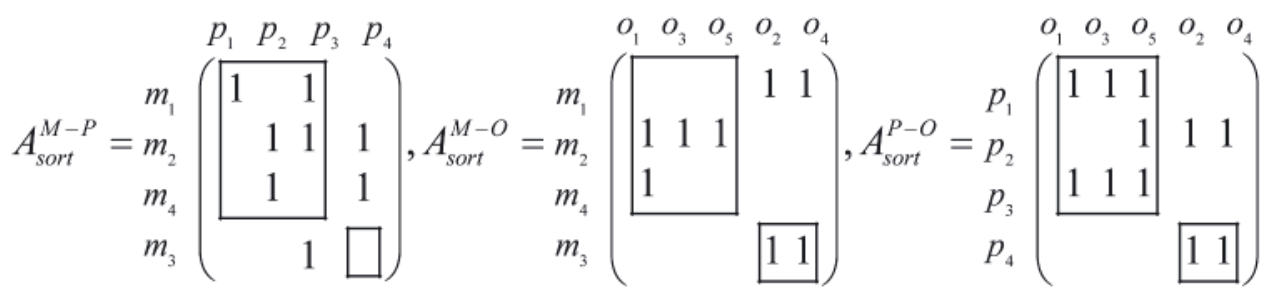

It can be hence stated that the proposed algorithm is potentially useful to grouping the sets whose incidences are expressed by binary variables. Certainly, quality of obtained results depends on assumed weights. Let's observe that the increasing of the weight q would cause the increasing of superiority of the solution obtained with Chebyshev measure over the result of Li. Simultaneously, the decreasing of the weight $\omega_{3}$ would result in improving the solution of Li and decreasing the quality of our solution.

\section{Example 2 - extension of the binary problem of $\mathrm{Li}$}

In order to present action of the grouping algorithm in the case of continuous incidence matrices the binary problem discussed in Example 1 will be modified. It is done by assigning random values from the interval $(0,1]$ to non-zero elements of incidence matrices. As a result the following problem is obtained:

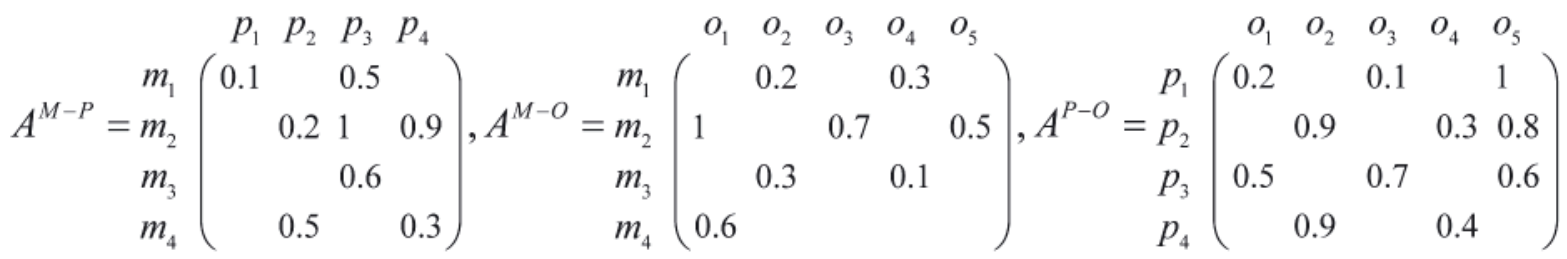

Tab. 3.2 presents the best results obtained with the use of the grouping algorithm. 
Tab. 3.2. Results of solving the problem ,Li” extended to continuous form

\begin{tabular}{|c|l|c|}
\hline Measure & \multicolumn{1}{|c|}{ Division } & $\begin{array}{c}\text { Quality } \\
\text { index }\end{array}$ \\
\hline \multirow{4}{*}{ Angular } & $\Gamma_{1}=\left(\left\{m_{1}\right\},\left\{p_{4}\right\},\left\{o_{1}\right\}\right)$, & \\
& $\Gamma_{2}=\left(\left\{m_{2}\right\},\left\{p_{1}\right\},\left\{o_{2}, o_{4}\right\}\right)$, & 0.379 \\
& $\Gamma_{3}=\left(\left\{m_{3}\right\},\left\{p_{3}\right\},\left\{o_{3}\right\}\right)$, & \\
& $\Gamma_{4}=\left(\left\{m_{4}\right\},\left\{p_{2}\right\},\left\{o_{5}\right\}\right)$ & \\
\hline \multirow{4}{*}{ Euclidean } & $\Gamma_{1}=\left(\left\{m_{1}\right\},\left\{p_{1}\right\},\left\{o_{1}\right\}\right)$, & \\
& $\Gamma_{2}=\left(\left\{m_{2}\right\},\left\{p_{2}\right\},\left\{o_{2}\right\}\right)$, & \\
& $\Gamma_{3}=\left(\left\{m_{3}\right\},\left\{p_{3}\right\},\left\{o_{3}\right\}\right)$, & \\
& $\Gamma_{4}=\left(\left\{m_{4}\right\},\left\{p_{4}\right\},\left\{o_{4}, o_{5}\right\}\right)$ & \\
\hline \multirow{4}{*}{ Manhattan } & $\Gamma_{1}=\left(\left\{m_{1}\right\},\left\{p_{1}, p_{3}\right\},\left\{o_{1}, o_{3}\right\}\right)$, & \\
& $\Gamma_{2}=\left(\left\{m_{2}, m_{4}\right\},\left\{p_{2}\right\},\left\{o_{2}\right\}\right)$, & 0.383 \\
& $\Gamma_{3}=\left(\left\{m_{3}\right\},\left\{p_{4}\right\},\left\{o_{4}, o_{5}\right\}\right)$ & \\
\hline \multirow{4}{*}{ Mahalanobis } & $\Gamma_{1}=\left(\left\{m_{3}\right\},\left\{p_{1}\right\},\left\{o_{1}, o_{3}\right\}\right)$, & \\
& $\Gamma_{2}=\left(\left\{m_{2}, m_{4}\right\},\left\{p_{2}\right\},\left\{o_{2}\right\}\right)$, & 0.406 \\
& $\Gamma_{3}=\left(\left\{m_{1}\right\},\left\{p_{3}, p_{4}\right\},\left\{o_{4}, o_{5}\right\}\right)$ & \\
\hline \multirow{5}{*}{ Chebyshev } & $\Gamma_{1}=\left(\left\{m_{1}\right\},\left\{p_{2}\right\},\left\{o_{2}\right\}\right)$, & \\
& $\Gamma_{2}=\left(\left\{m_{4}\right\},\left\{p_{1}\right\},\left\{o_{4}\right\}\right)$, & \\
& $\Gamma_{3}=\left(\left\{m_{2}\right\},\left\{p_{3}\right\},\left\{o_{1}\right\}\right)$, \\
& $\Gamma_{4}=\left(\left\{m_{3}\right\},\left\{p_{4}\right\},\left\{o_{3}, o_{5}\right\}\right)$ & $\mathbf{0 . 5 3 6}$ \\
\hline & & \\
\hline
\end{tabular}

Also in this case the Chebyshev measure turns out to be most effective in grouping the elements. This time the division into four specialized groups is the best. In matrix form it can be presented as follows:

\subsection{Example 3 - practical problem - the welding of steel structures}

\subsubsection{Problem description}

Let an example of building five kinds of input structures shown in Fig. 3.1, be considered. The structures have been not subjected to any analysis of possible, at least partial, unification of their sets of lower-stage subassemblies. Intentionally, real rough data are considered.
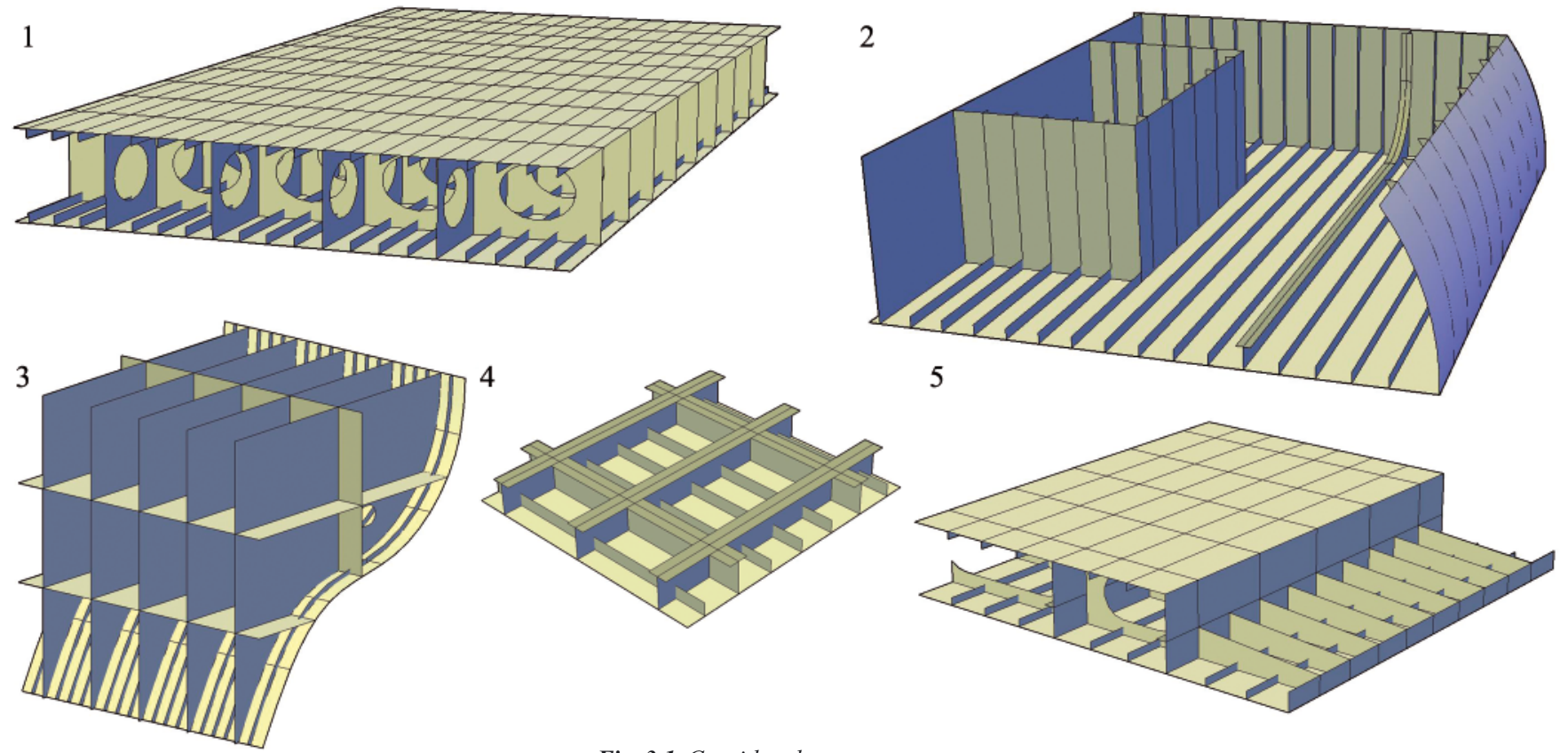

Fig. 3.1. Considered output structures 
On the basis of technical documentation of subdivision of the output structures into prefabrication steps, a list of 37 input structures (subassemblies) was elaborated. They will be joined together by welding processes. The continuous incidence matrix $\mathrm{A}^{\mathrm{C}^{\mathrm{in}}-\mathrm{C}^{\mathrm{Out}}}$, after its normalization within the interval $[0,1]$, takes the following form:

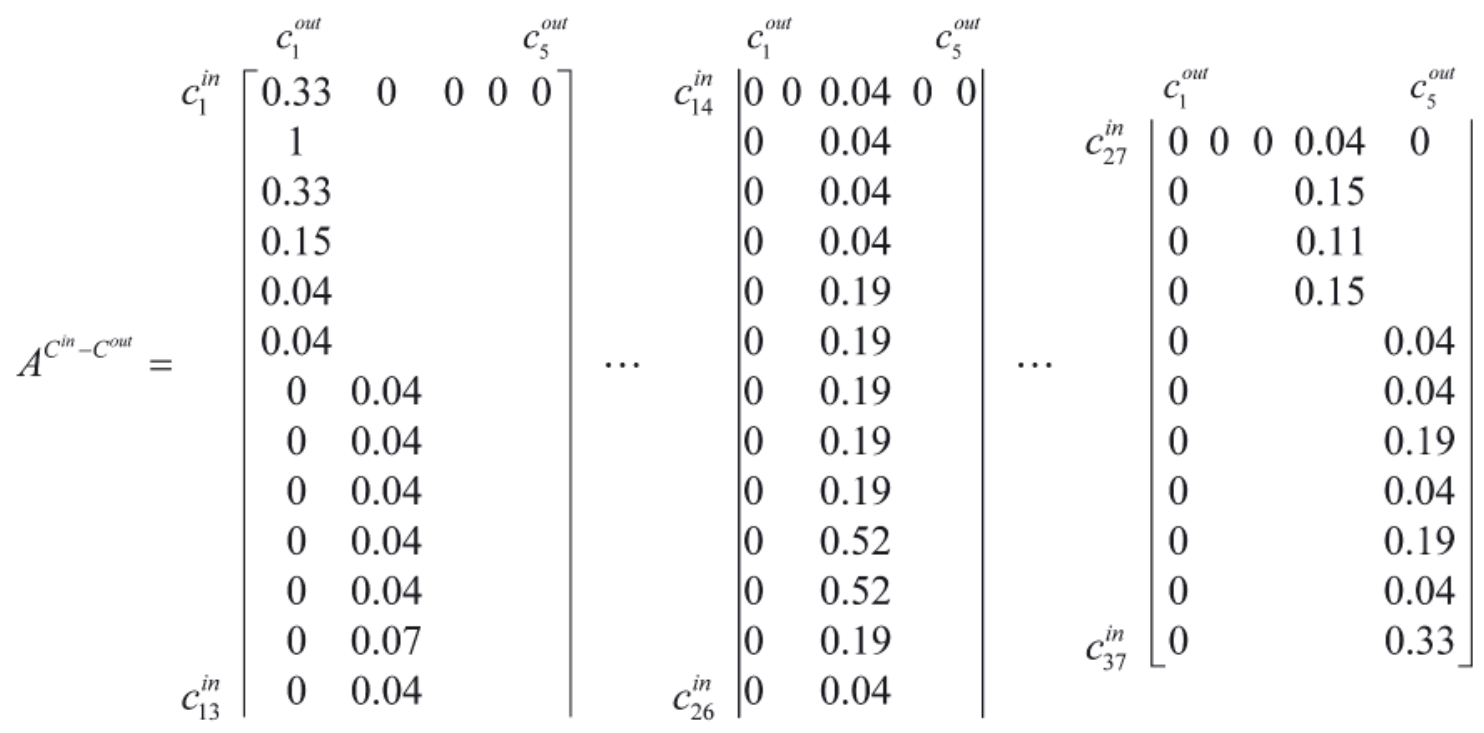

The third set of elements is formed from welding operations which differ to each other as regards welding position (Fig. 3.2), type of weld ( B - butt, $\mathrm{T}$ - fillet) and accessiblity for welding machine ( $\mathrm{M}$ - semi-automatic, $\mathrm{R}$ - automatic).

Not all combinations of the features take place hence the set $S$ has eight elements only - see Tab. 3.3.

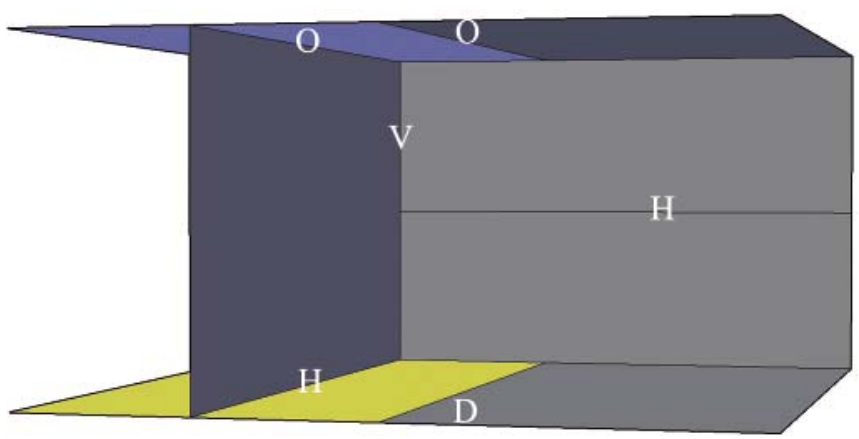

Fig. 3.2. Welding positions: $O$-overhead, $V$-vertical, $H$-overside, horizontal, $D$ - floor

Tab. 3.3. Symbols of welding operation types

\begin{tabular}{|c|c|c|c|}
\hline $\begin{array}{c}\text { Element of } \\
\text { the set } \mathbf{S}\end{array}$ & Position & Type of joint & $\begin{array}{c}\text { Degree of } \\
\text { automation }\end{array}$ \\
\hline $\mathrm{s}_{1}$ & $\mathrm{O}$ & $\mathrm{T}$ & $\mathrm{M}$ \\
\hline $\mathrm{s}_{2}$ & $\mathrm{O}$ & $\mathrm{T}$ & $\mathrm{R}$ \\
\hline $\mathrm{s}_{3}$ & $\mathrm{~V}$ & $\mathrm{~T}$ & $\mathrm{M}$ \\
\hline $\mathrm{s}_{4}$ & $\mathrm{~V}$ & $\mathrm{~T}$ & $\mathrm{R}$ \\
\hline $\mathrm{s}_{5}$ & $\mathrm{H}$ & $\mathrm{B}$ & $\mathrm{R}$ \\
\hline $\mathrm{s}_{6}$ & $\mathrm{H}$ & $\mathrm{T}$ & $\mathrm{M}$ \\
\hline $\mathrm{s}_{7}$ & $\mathrm{H}$ & $\mathrm{T}$ & $\mathrm{R}$ \\
\hline $\mathrm{s}_{8}$ & $\mathrm{D}$ & $\mathrm{B}$ & $\mathrm{R}$ \\
\hline
\end{tabular}

On the basis of an analysis of output structures, lengths of the welds made in the distinguished welding positions, were determined. Also, for each of the input structures lengths of the welds for its joining with other input structures, were determined. As a result the two successive normalized incidence matrices were obtained:

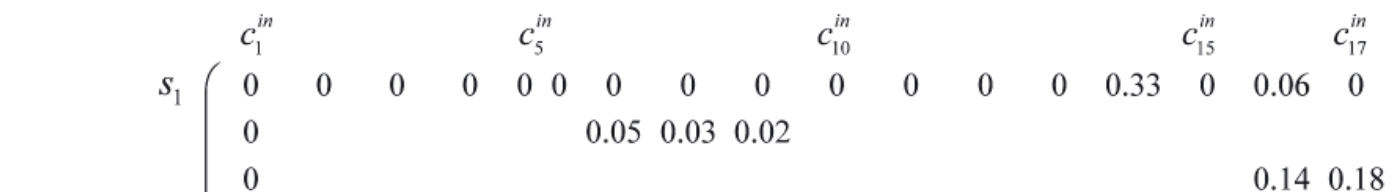

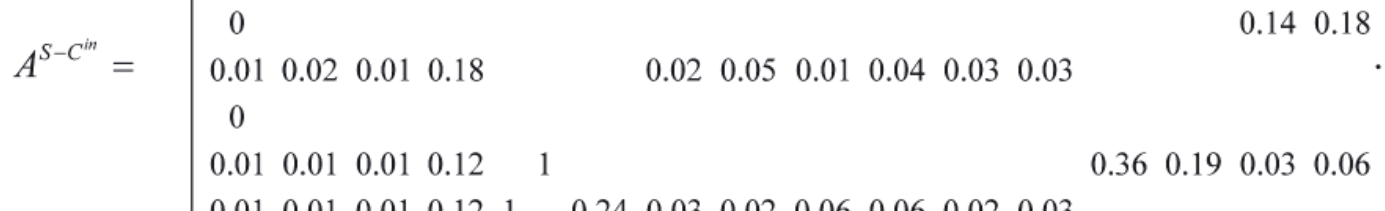

$$
\begin{aligned}
& s_{8}\left(\begin{array}{cccccccccccc}
0.01 & 0.01 & 0.01 & 0.12 & 1 & 0.24 & 0.03 & 0.02 & 0.06 & 0.06 & 0.02 & 0.03 \\
0 & & & & & & & & & & &
\end{array}\right.
\end{aligned}
$$

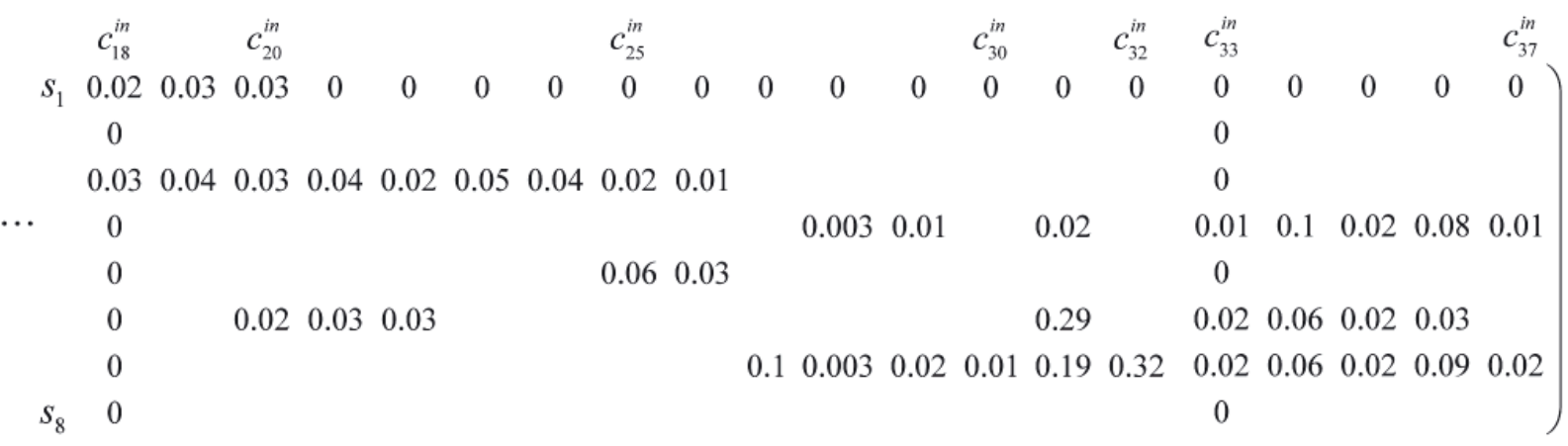




$$
A^{S-C^{\text {out }}}=S_{1}\left(\begin{array}{ccccc}
c_{1}^{\text {out }} & & & \multicolumn{2}{c}{c_{5}^{\text {out }}} \\
0 & 0 & 0.17 & 0 & 0 \\
0 & 0.02 & & & \\
0 & & 1 & & \\
0.29 & 0.04 & & 0.01 & 0.08 \\
0 & & 0.06 & & \\
0.41 & & 0.2 & & 0.13 \\
0.41 & 0.1 & & 0.04 & 0.2 \\
0 & & & 0.004 &
\end{array}\right)
$$

\subsubsection{Results of the grouping}

With the use of the algorithm described in Chapter 2.3 the divisions into 2, 3, 4 and 5 three-dimensional groups were obtained according to the condition. The following values of weighing coefficients: $\mathrm{q}=0.5, \omega_{\mathrm{S}-\mathrm{C}_{\text {in }}}=\omega_{\mathrm{S}-\mathrm{C}_{\text {out }}}=\omega_{\mathrm{C}_{\text {in }}-\mathrm{C}_{\text {out }}}=1$ were assumed.

Five measures of distance between elements were tested, i.e. the angular, Manhattan, Euclidean, Chebyshev and Mahalanobis one. For four numbers of groups and five distance measures, twenty divisions and quality indices corresponding to them, were obtained - Fig. 3.3.

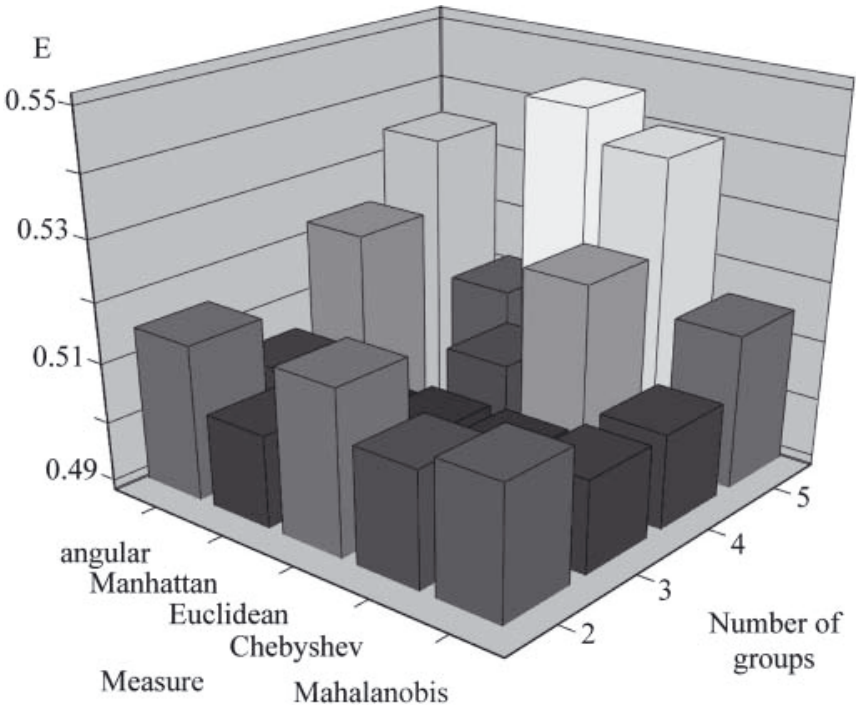

Fig. 3.3. Quality indices of divisions for different numbers of groups and distance measures

The best solution was obtained for the division into five groups by using Euclidean measure: $(\mathrm{E}(\Gamma)=0.545)$. The groups are as follows:

$$
\begin{aligned}
\Gamma_{1}= & \left(\left\{s_{5}\right\},\left\{c_{5}^{\text {in }}, c_{6}^{\text {in }}, c_{31}^{\text {in }}\right\},\left\{c_{5}^{\text {out }}\right\}\right) \\
\Gamma_{2}= & \left(\left\{s_{8}\right\},\left\{c_{1}^{\text {in }}, c_{2}^{\text {in }}, c_{3}^{\text {in }}, c_{7}^{\text {in }}, \ldots, c_{13}^{\text {in }}, c_{18}^{\text {in }}, c_{19}^{\text {in }},\right.\right. \\
& \left.\left.c_{21}^{\text {in }}, \ldots, c_{30}^{\text {in }}, c_{32}^{\text {in }}, \ldots,, c_{37}^{\text {in }}\right\},\left\{c_{4}^{\text {out }}\right\}\right) \\
\Gamma_{3}= & \left(\left\{s_{1}, s_{3}\right\},\left\{c_{17}^{\text {in }}\right\},\left\{c_{3}^{\text {out }}\right\}\right) \\
\Gamma_{4}= & \left(\left\{s_{4}, s_{6}, s_{7}\right\},\left\{c_{4}^{\text {in }}\right\},\left\{c_{1}^{\text {out }}\right\}\right) \\
\Gamma_{5}= & \left(\left\{s_{2}\right\},\left\{c_{14}^{\text {in }}, c_{15}^{\text {in }}, c_{16}^{\text {in }}, c_{20}^{\text {in }}\right\},\left\{c_{2}^{\text {out }}\right\}\right)
\end{aligned}
$$

If the grouping between pairs of the sets are taken into account then it can be observed that the greatest mean value in the groups (Eq. ) is always reached for the division into five groups. However effectiveness of the measures shows different values for different pairs, e.g. for:

- $\mathrm{S}-\mathrm{C}^{\text {in }}$ - angular measure is the best, $\left(\eta_{1}\left(\mathrm{~A}^{\mathrm{s}}-\mathrm{C}^{\mathrm{in}}, \Gamma\right)=\right.$ $=0.0515)$

- $\mathrm{S}-\mathrm{C}^{\text {in }}-$ Euclidean measure is the best $\left(\eta_{1}\left(\mathrm{~A}^{\mathrm{S}}-\mathrm{C}^{\text {out }}, \Gamma\right)=\right.$ $=0.2857$ ),

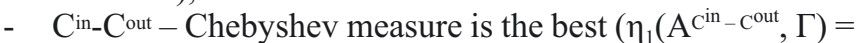
$=0.1131)$.

As can be observed, during analyzing we are forced to take into account various measures, since their effectiveness depends on values of co-ordinates of grouped elements.

In the above discussed example can be observed a tendency for breaking up the division into as many groups as possible. It results from a low density of incidence matrices which constitute input data. And, such density directly results from the breaking-up of the set of input structures. Reduction in size of the set $\mathrm{C}$ in is possible by standardizing the input structures. Influence of such approach on results of grouping will be discussed in a separate publication.

\section{CONCLUSIONS}

In this paper has been presented the algorithm which on the basis of three assumed sets, groups their elements in such a way as locate at least one element out of each set into each of the group. In the algorithm the method of cluster analysis of $\mathrm{k}$-means as well as five different measures of distance between multi-dimensional space points, was applied. It was elaborated with the aim of its applications to organization of production of $3 \mathrm{~S}$ structures. To this end, were distinguished three sets of elements occurring in enterprises which produce structures of the kind. The sets as well as continuous matrices of incidence between them form input data for the proposed grouping algorithm. These are: set of working cells, set of input structures (subassemblies) and set of output structures.

The grouping division obtained this way can find application during designing production cells. Certainly, the above determined groups are only an indication as to mutual location of working cells and their equipment. Three-dimensional group can be interpreted as an indication for very close location of:

- working cells for production of concrete input structures

(or points of delivery of the structures from outside of the enterprise),

- working cells for joining the structures into larger objects (output structures) as well as

- working cells which use the output structures for further joining them, or possible cleaning, painting, fitting, launching or also loading and dispatching.

Full modification of a non-optimum spatial organization of working cells may be impossible. It is hard to expect that because of results of grouping whole workshop buildings would be moved. However enterprise logistic system is much more adaptable in this respect.

The group may be hence interpreted also as an indication for elevation of enterprise investment priorities in certain areas.

It can be:

- extension of internal roads between selected cells;

- purchase of transport means dedicated to certain structures, acc. their mass, gabarites, shapes;

- ordering, to subcontractors, some workings which especially disturb determined production flows within enterprise. 
A general conclusion which can be drawn on the basis of the performed calculations is that the problem of designing group technologies becomes greatly complicated in the moment of relating it to real production problems. Theoretical methods in this area have been developed for tens of years however reality seriously challenges both theoreticians and engineers aimed at implementing the methods into practice.

\section{BIBLIOGRAPHY}

1. Ballakur A., Steudel H.J.: A within-cell utilization based heuristic for designing cellular manufacturing systems. International Journal of Production Research, 25, 1987, 639665.

2. Ben-Arieh D., Sreenivasav R.: Information analysis in a distributed dynamic group technology method. International Journal of Production Economics 60-61, 1999, 427-432.

3. Bhat M.V., Haupt A.: An efficient clustering algorithm. IEEE transactions on systems. Man and Cybernetics SMC-6 (1), 1976, 61-64.

4. Boctor F.F.: A linear formulation of the machine-part cell formation problem. International Journal of Production Research, 29 (2), 1991, 343-356.

5. Boe W.J., Cheng C.H.: A close neighbor algorithm for designing a cellular manufacturing system. International Journal of Production Research, 29 (10), 1991, 2097-2116.

6. Chan H.M., Milner D.A.: Direct clustering algorithm for group formation in cellular manufacture. Journal of Manufacturing Systems, 1 (1), 1982, 65-75.

7. Chandrasekharan M.P., Rajagopalan R.: An ideal seed nonhierarchical clustering algorithm for cellular manufacturing. International Journal of Production Research, 24 (2), 1986,451464

8. Cho K.-K., Sun J.-G., Oh J.-S.: An automated welding operation planning system for block assembly in shipbuilding. International Journal of Production Economics 60-61 (1), 1999, 203-209.

9. Groover M.P.: Fundamentals of modern manufacturing: materials, processes and systems. 4th ed., John Wiley\&Sons, 2010
10.Hartigan J. A.: Clustering Algorithms. John Wiley \& Sons Inc., 1975.

11.Hassan M.M.D.: Layout design in group technology manufacturing. International Journal of Production Economics, 38, 1995. 173-188.

12.Iwańkowicz R.: Object-matrix model of complex manufacturing technology. Industrial Management \& Data Systems, 108 (8), 2008, 1131-1148.

13.Kitaoka M., Nakamura, R., Serizawa, S., Usuki, J.: Multivariate analysis model for machine-part cell formation problem in group technology. International Journal of Production Economics, 60-61, 1999, 433-438.

14.Li M.-L.: The algorithm for integrating all incidence matrices in multi-dimensional group technology. International Journal of Production Economics, 86, 2003, 121-131.

15.Li M.-L.: Efficiency measurement for multi-dimensional group technology. International Journal of Advanced Manufacturing Technology, 35, 2007, 621-632.

16.Li M.-L., Parkin R.E.: Group technology revisited: a simple and robust algorithm with enhanced capability. International Journal of Production Research, 35 (7), 1997, 1969-1992.

17.Min H., Shin D.: Simultaneous formation of machine and human cells in group technology: A multiple objective approach International Journal of Production Research, 31 (1), 1993, $2307-2318$.

18.Parkin R.E., Li M.-L.: The multi-dimensional aspects of a group technology algorithm. International Journal of Production Research, 35 (8), 1997, 2345-2358.

19.Tan P.-N., Steinbach M., Kumar V.: Introduction to Data Mining. Addison Wesley, 2005, 496-515.

\section{CONTACT WITH THE AUTHOR}

Remigiusz Iwańkowicz, Ph.D.

West Pomeranian University of Technology, Szczecin Faculty of Marine Technology and Transport

Al. Piastów 41

71-065 Szczecin, POLAND

e-mail: iwankow@zut.edu.pl 